IMSC/2004/11/38

hep-th/0412033

\title{
Loop Variables and the (Free) Open String in a Curved Background.
}

\author{
B. Sathiapalan \\ Institute of Mathematical Sciences \\ Taramani \\ Chennai, India 600113 \\ bala@imsc.res.in
}

November 1, 2018

\begin{abstract}
Using the loop variable formalism as applied to a sigma model in curved target space, we give a systematic method for writing down gauge and generally covariant equations of motion for the modes of the free open string in curved space. The equations are obtained by covariantizing the flat space equation and then demanding gauge invariance, which introduces additional curvature couplings. As an illustration of the procedure, the spin two case is worked out explicitly.
\end{abstract}




\section{Introduction}

The loop variable approach is a proposal for writing down gauge invariant equations of motion for the string. It has been applied to both the open and closed bosonic string theories and interacting gauge invariant equations have been written down. As it is based on the sigma model renormalization group $[1,2,3,4]$ there is the possibility of addressing the issue of manifest background independence in string theory [22]. We thus try and generalize the loop variable construction to curved space time backgrounds. As a first step we do this for the free open string. We give a prescription for writing down gauge invariant and generally covariant equations of motion for all the modes of the string generalizing the results of [5] to curved space. We first do a straightforward covariantization of the open string equations of motion. This violates gauge invariance. We show that gauge invariance can be restored by adding curvature couplings. These can be obtained systematically. We give the general procedure and illustrate it with the simplest non-trivial case, which is spin-2. The spin-2 equations in curved space can also be obtained by Kaluza-Klein reduction of Einstein gravity in one higher dimension [6]. The loop variable method however can be just as easily applied to arbitrarily high spin fields.

In principle one should be able to derive all the couplings to the curvature tensor by including the interaction of the bulk and boundary in the sigma model approach and if done correctly, the resulting equations should be gauge invariant. We have not done this in this paper. We have obtained the couplings just by requiring gauge invariance. Assuming that there is a unique gauge invariant and generally covariant equation, we should get the same answer both ways. However it would be interesting to explicitly check this.

In the loop variable approach the self-interactions can usually be obtained by thickening the loop to a band $[7,8,9]$. We expect this will be the case here also, although we have not addressed this issue in this paper.

The problems that one faces when one tries to write higher spin equations in curved space has been the subject of much investigation and solutions have also been given $[10,11,12,13,14,15]$. It would be interesting to find the relation between these results and the present work which is in the context of string theory.

This paper is organized as follows. In Section 2 we briefly review the sigma model in curved target space and the Riemann Normal Coordinate expansion. In Section 3 we apply it to the loop variable to get covariant 
equations of motion and explain how to modify them to make them gauge invariant. Section 4 contains conclusions.

\section{Sigma Model in Curved Space}

\subsection{Riemann Normal Coordinates}

In this section we remind the reader about Normal coordinates [19]. Let $x^{\mu}$ be a coordinate system with the notation $x^{\mu}(P)=x^{\mu}$ where $P$ denotes a generic point of the manifold, and let $x_{0}^{\mu}$ be the coordinates of a point $P_{0}$ in the manifold, at which we define Riemann Normal Coordinates (RNC). In the RNC the location of a generic point $P$ is described by a set of real numbers $y^{\mu}$ that are defined by the following: Consider the geodesic $x^{\mu}(s)$ that goes through $P_{0}$ as well as $P$. The parameter $s$ can be chosen to be equal to the distance along the geodesic. So $x^{\mu}(0)=x_{0}^{\mu}$. Let the unit tangent vector to this geodesic at $P_{0}$ be $\xi^{\mu}$. Thus

$$
\xi^{\mu}=\left.\frac{d x^{\mu}}{d s}\right|_{x_{0}}
$$

If $s$ is the distance to point $P$ then $y^{\mu}=s \xi^{\mu}$ defines coordinates of the point $P$. While the tangent vector to the geodesic at $P_{0}$ is a geometric object the components $\xi^{\mu}$ defined above are with respect to the original $x$ coordinate axes. One can equally well choose any other basis in the tangent space at $x_{0}$ to define the components. Thus the RNC and the vectors $\xi^{\mu}$ are independent of our original coordinate system $x^{\mu}$. But $x$ can be expressed as a (in general nonlinear) function of $y, x(y)$, with $x(0)=x_{0}$. $\xi$ and hence $y$ is a contravariant vector defined at the point $P_{0}$, and so if we change our basis in the tangent space the vectors transform contravariantly. In particular if we use (2.1) to define the components, then the change in coordinate from $x$ to $x^{\prime}$, will change $y^{\prime \mu}=\left.\left(\frac{\partial x^{\prime \mu}}{\partial x^{\nu}}\right)\right|_{x_{0}} y^{\nu}=a_{\nu}^{\mu} y^{\nu}$. Here the matrix $a_{\nu}^{\mu}$ are a set of numbers.

An immediate consequence of all this is the following. Consider the Taylor expansion of a tensor field at the point $x(y)$ about it's value at the point $x(y=0)=x_{0}$ :

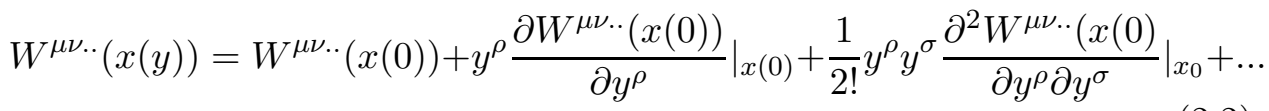


This expansion is valid in any coordinate system but if we let $x$ be a RNC we have some simplification. Let $x^{\mu}=x_{0}^{\mu}+y^{\mu}$ exactly. In this coordinate system the geodesics at $P_{0}$ look like straight lines. Thus $\Gamma_{\nu \rho}^{\mu}\left(x_{0}\right)=0$, although the derivatives of $\Gamma$ are not zero. The derivatives obey

$$
\partial_{\left(\mu_{1}\right.} \Gamma_{\rho \sigma)}^{\nu}=\partial_{\left(\mu_{1} \mu_{2}\right.} \Gamma_{\rho \sigma)}^{\nu}=\partial_{\left(\mu_{1} \mu_{2} \ldots \mu_{r}\right.} \Gamma_{\rho \sigma)}^{\nu}=0
$$

Thus for instance using $\Gamma\left(x_{0}\right)=0$ and $(2.3)$ and also,

$$
R_{\rho \mu \sigma}^{\nu}\left(x_{0}\right)=\partial_{\mu} \Gamma_{\rho \sigma}^{\nu}-\partial_{\sigma} \Gamma_{\rho \mu}^{\nu}
$$

we get

$$
\partial_{\mu} \Gamma_{\rho \sigma}^{\nu}\left(x_{0}\right)=\frac{1}{3}\left(R_{\rho \mu \sigma}^{\nu}\left(x_{0}\right)+R_{\sigma \mu \rho}^{\nu}\left(x_{0}\right)\right)
$$

Using these results one can write down relations of the following type:

$$
\begin{gathered}
\partial_{\mu} W_{\alpha}\left(x_{0}\right)=D_{\mu} W_{\alpha}\left(x_{0}\right)+\Gamma_{\alpha \mu}^{\beta} W_{\beta}\left(x_{0}\right)=D_{\mu} W_{\alpha}\left(x_{0}\right) \\
\partial_{\nu} \partial_{\mu} W_{\alpha}\left(x_{0}\right)=\left(\partial_{\nu}\left(D_{\mu} W_{\alpha}\left(x_{0}\right)+\Gamma_{\alpha \mu}^{\beta} W_{\beta}\left(x_{0}\right)\right)=D_{\nu} D_{\mu} W_{\alpha}+\left(\partial_{\nu} \Gamma_{\alpha \mu}^{\beta}\right) W_{\beta}\left(x_{0}\right)\right. \\
=D_{\nu} D_{\mu} W_{\alpha}+\frac{1}{3}\left(R_{\alpha \nu \mu}^{\beta}+R_{\mu \nu \alpha}^{\beta}\right) W_{\beta}
\end{gathered}
$$

and more generally one can write a "covariant" Taylor expansion [19]:

$$
\begin{gathered}
W_{\alpha_{1} \ldots \alpha_{p}}(x)=W_{\alpha_{1} \ldots \alpha_{p}}\left(x_{0}\right)+W_{\alpha_{1} \ldots \alpha_{p}, \mu}\left(x_{0}\right) y^{\mu}+ \\
\frac{1}{2 !}\left\{W_{\alpha_{1} \ldots \alpha_{p}, \mu \nu}\left(x_{0}\right)-\frac{1}{3} \sum_{k=1}^{p} R_{\mu \alpha_{k} \nu}^{\beta}\left(x_{0}\right) W_{\alpha_{1} . . \alpha_{k-1} \beta \alpha_{k+1} . . \alpha_{p}}\left(x_{0}\right)\right\} y^{\mu} y^{\nu}+ \\
\frac{1}{3 !}\left\{W_{\alpha_{1} \ldots \alpha_{p}, \mu \nu \rho}\left(x_{0}\right)-\sum_{k=1}^{p} R_{\mu \alpha_{k} \nu}^{\beta}\left(x_{0}\right) W_{\alpha_{1} \ldots \alpha_{k-1} \beta \alpha_{k+1} . . \alpha_{p}, \rho}\left(x_{0}\right)\right. \\
\left.-\frac{1}{2} \sum_{k=1}^{p} R_{\mu \alpha_{k} \nu, \rho}^{\beta}\left(x_{0}\right) W_{\alpha_{1} . . \alpha_{k-1} \beta \alpha_{k+1} . . \alpha_{p}}\left(x_{0}\right)\right\} y^{\mu} y^{\nu} y^{\rho}+\ldots
\end{gathered}
$$

Thus in the RNC we see that each term in the Taylor expansion is manifestly a tensor (defined at $x_{0}$ ) and thus the sum is a tensor at the point $x_{0}$. Thus we see that the Taylor expansion, while valid in any coordinate system, becomes in the RNC, a sum of tensors. However the RHS is a tensor at $x_{0}$ and the LHS is a tensor at $x$. Thus this equation (2.7) is not a covariant tensor equation. It holds only in the RNC. 


\subsection{Sigma Model with Curved Target Space}

Following [20, 21] we can consider a sigma model in curved background, with bulk action:

$$
S[X]=-\frac{1}{2} \int d^{2} \sigma G_{\mu \nu}(X) \partial_{\alpha} X^{\mu}(\sigma, \tau) \partial_{\alpha} X^{\nu}(\sigma, \tau)
$$

We use RNC coordinates $y^{\mu}$ and Taylor expand all the tensors. Thus $G_{\mu \nu}(X)=G_{\mu \nu}\left(x_{0}\right)+O\left(y^{\mu}\right)$. We neglect the higher order terms. As stated in the introduction these are responsible for closed string - open string interactions beyond those that are obtained by covariantizing derivatives. We are going to obtain these by requiring gauge invariance. We choose a RNC

$$
X^{\mu}(\sigma, \tau)=x_{0}^{\mu}+y^{\mu}(\sigma, \tau)
$$

where $x_{0}$ is a constant (independent of $\left.\sigma, \tau\right)$ and is the reference point of our RNC. The kinetic term for $y^{\mu}$ is $G_{\mu \nu}\left(x_{0}\right) \partial_{\alpha} y^{\mu} \partial^{\alpha} y^{\nu}$. Generalizing the flat case prescription [5], we set for the coincident propagator:

$$
<y^{\mu}\left(\sigma^{\alpha}\right) y^{\nu}\left(\sigma^{\alpha}\right)>=G^{\mu \nu}\left(x_{0}\right) \rho\left(\sigma^{\alpha}\right)
$$

where $\rho$ is the Liouville mode of the world sheet.

We now consider the loop variable $(\mathrm{LV}) e^{i \sum_{n \geq 0} k_{n} Y_{n}(z)}$, obtained as usual by the Taylor expansion of $e^{i \int_{c} \alpha(s) k(s) \partial_{z} y(z+s) d s+i k_{0} y(z)}$, where

$$
Y=y+\alpha_{1} \partial_{z} y+\alpha_{2} \partial_{z}^{2} y+\alpha_{3} \frac{\partial^{3} z}{2 !}+\ldots
$$

and $\alpha_{n}$ are the modes of $\alpha .{ }^{1}$ Correspondingly, again generalizing [5], we have

$$
<Y^{\mu}\left(\sigma^{\alpha}\right) Y^{\nu}\left(\sigma^{\alpha}\right)>=G^{\mu \nu}\left(x_{0}\right) \Sigma\left(\sigma^{\alpha}\right)
$$

Apart from the presence of the background metric, there is no difference with the flat space case. Thus the equations of motion are obtained by requiring

$$
\frac{\delta}{\delta \Sigma}: e^{i \sum_{n \geq 0} k_{n} Y_{n}(z)}: e^{\sum_{n, m \geq 0} k_{n} \cdot k_{m} \frac{1}{2}\left(\frac{\partial^{2}}{\partial x_{n} \partial x_{m}}-\frac{\partial}{\partial x_{n+m}}\right) \Sigma}=0
$$

\footnotetext{
${ }^{1}$ These are all described in $[5,7]$. We refer the reader to those papers. A short summary is given in the Appendix.
} 
Here $k_{0} . y=k_{\mu} y^{\mu}$ and all space time indices are contracted using $G_{\mu \nu}\left(x_{0}\right)$. $k_{0 \mu}$ will thus become $-i \frac{\partial}{\partial y^{\mu}}$. The change

$$
k_{n} \rightarrow k_{n}+\lambda_{p} k_{n-p}
$$

changes the loop variable $(2.13)$ by a total derivative $\frac{\partial}{\partial x_{p}}$ and leaves the equations obtained from it invariant. These are the gauge transformations.

\section{Application to Loop Variables: Covariant Equa- tions of Motion}

We can now proceed in the usual way to get the equations of motion in terms of loop variables [5]. The difference with the flat space case will show up when we map loop variables to space time fields since $k_{0}^{\mu}$ will be mapped to covariant derivatives as described in the previous paragraph. Thus we will obtain a covariant equation in terms of tensors at the point $x_{0}$. Since $x_{0}$ is arbitrary, we can take this as the (covariant) equations of motion that we are after. The second step is to obtain the gauge transformation in terms of space time fields.

\subsection{Covariantizing the Equations}

We give below, the loop variable equation and the corresponding covariant equation for each of the fields.

\section{Tachyon:}

The equation for the tachyon is:

$$
\left[k_{0}^{\mu} k_{0 \mu}+k_{0}^{5} k_{0}^{5}\right]=0
$$

In flat space (or in RNC) this becomes (on setting $\left(k_{0}^{5}\right)^{2}=\operatorname{dim}[V]-1$, where $\operatorname{dim}[V]$ is the world sheet dimension of the vertex operator corresponding to that mode [5]) :

$$
\left(-\partial_{\mu} \partial^{\mu}-1\right) \Phi(x)=0
$$

The covariant equation is therefore:

$$
\left(-D_{\mu} D^{\mu}-1\right) \Phi(x)=0
$$

There is no gauge transformation for the tachyon. 


\section{Photon:}

The loop variable equation for the vector (photon) is

$$
\left[k_{0}^{\mu} k_{0 \mu} k_{1}^{\nu}-k_{0}^{\mu} k_{1 \mu} k_{0}^{\nu}\right]=0
$$

Where

$$
<k_{1}^{\mu}>=A^{\mu} .
$$

In RNC this is Maxwell's equation:

$$
\partial^{\mu} \partial_{[\mu} A_{\nu]}(x)=0
$$

Using (2.6) we get in curved space:

$$
D^{\mu}\left[\partial_{\mu} A_{\nu}-\partial_{\nu} A_{\mu}\right]=0
$$

The gauge transformation

$$
k_{1}^{\mu} \rightarrow k_{1}^{\mu}+\lambda_{1} k_{0}^{\mu}
$$

leaves the LV equation invariant. Setting $\left\langle\lambda_{1}\right\rangle=\Lambda_{1}\left(k_{0}\right)$, gives us the gauge transformation $A_{\mu} \rightarrow A_{\mu}+\partial_{\mu} \Lambda$. Since $\Lambda$ is a scalar, this is unmodified in curved space and continues to be an invariance of Maxwelll's equation in curved space (3.6).

\section{Spin 2:}

There are three fields $\left\langle k_{1}^{\mu} k_{1}^{\nu}>=-S_{1,1}^{\mu \nu},<k_{2}^{\mu}>=-i S_{2}^{\mu}\right.$ and $<k_{2}^{5}>=$ $S_{2}^{5}$. The last two are auxiliary fields that are necessary for gauge invariance. We also have to make the identifications $k_{1}^{\mu} k_{1}^{5}=k_{2}^{\mu} k_{0}^{5}$ and $k_{1}^{5} k_{1}^{5}=k_{2}^{5} k_{0}^{5}$. As explained in [5] this is necessary in order to reproduce the string spectrum. Furthermore $k_{0}^{5}=1$ because the spin two vertex operators have dimension 2.

The loop variable equation for spin-2 is obtained by equating the coefficients of $\frac{\partial}{\partial x_{1}} Y^{\mu} \frac{\partial}{\partial x_{1}} Y^{\nu}, \frac{\partial}{\partial x_{2}} Y^{\nu}, \frac{\partial}{\partial x_{1}} Y^{5} \frac{\partial}{\partial x_{1}} Y^{5}$ in (2.13), to zero [5]:

$$
\begin{aligned}
&-\left(k_{0}^{2}+\left(k_{0}^{5}\right)^{2}\right) k_{1}^{\mu} k_{1}^{\nu}+k_{1}^{(\mu} k_{0}^{\nu)} k_{1} \cdot k_{0}- \\
& k_{0}^{\mu} k_{0}^{\nu} k_{1} \cdot k_{1}+k_{1}^{(\mu} k_{0}^{\nu)} k_{1}^{5} k_{0}^{5}-k_{1}^{5} k_{1}^{5} k_{0}^{\mu} k_{0}^{\nu}=0 \\
& k_{0}^{2} k_{2}^{\mu}-k_{1}^{\mu} k_{1} \cdot k_{0}+k_{0}^{\mu} k_{1} \cdot k_{1}-k_{0}^{\mu} k_{2} \cdot k_{0}=0 \\
&-\left(k_{1} \cdot k_{0}\right)^{2}+\left(k_{0}^{2}+\left(k_{0}^{5}\right)^{2}\right) k_{1} \cdot k_{1}-2 k_{1}^{5} k_{0}^{5} k_{1} \cdot k_{0}+k_{0}^{2} k_{1}^{5} k_{1}^{5}=0
\end{aligned}
$$


The LV equation is invariant under

$$
k_{2}^{\mu} \rightarrow k_{2}^{\mu}+\lambda_{1} k_{1}^{\mu}+\lambda_{2} k_{0}^{\mu} \quad, \quad k_{1}^{\mu} \rightarrow k_{1}^{\mu}+\lambda_{1} k_{0}^{\mu}
$$

In RNC the equation for $S_{1,1}^{\mu \nu}$ is:

$$
\left(\partial_{\rho} \partial^{\rho}-\left(k_{0}^{5}\right)^{2}\right) S_{1,1}^{\mu \nu}-\partial_{\rho} \partial^{(\mu} S_{1,1}^{\nu) \rho}+\partial^{\mu} \partial^{\nu} S_{1,1 \rho}^{\rho}+\partial^{(\mu} S_{2}^{\nu)}\left(k_{0}^{5}\right)^{2}-\partial^{\mu} \partial^{\nu} S_{2} k_{0}^{5}=0
$$

$k_{0}^{5}=1$, but we leave it as it is for later convenience.

We can now "covariantize" this equation. Using a generalization of (2.6) we get

$$
\partial_{\rho} \partial_{\sigma} S_{\mu \nu}=D_{\rho} D_{\sigma} S_{\mu \nu}+\frac{1}{3}\left(R_{\mu \rho \sigma}^{\alpha}+R_{\sigma \rho \mu}^{\alpha}\right) S_{\alpha \nu}+\frac{1}{3}\left(R_{\nu \rho \sigma}^{\alpha}+R_{\sigma \rho \nu}^{\alpha}\right) S_{\mu \alpha}
$$

Making use of the above one finds the covariant equation:

$$
\begin{gathered}
D^{\rho} D_{\rho} S_{\mu \nu}-\left(k_{0}^{5}\right)^{2} S_{\mu \nu} \\
-\left[D^{\rho} D_{\nu} S_{\mu \rho}+D^{\rho} D_{\mu} S_{\nu \rho}+\frac{2}{3}\left(R_{\mu \nu}^{\alpha \rho}+R_{\nu}^{\alpha \rho}{ }_{\mu}\right) S_{\alpha \rho}\right] \\
+D_{\mu} D_{\nu} S_{1,1 \rho}^{\rho}+\left(D_{\mu} S_{2 \nu}+D_{\nu} S_{2 \mu}\right)\left(k_{0}^{5}\right)^{2}-D_{\mu} D_{\nu} S_{2} k_{0}^{5}=0 .
\end{gathered}
$$

The gauge transformation (3.11) in RNC reads

$$
\delta S_{1,1 \mu \nu}=\partial_{(\mu} \Lambda_{1,1 \nu)} \quad, \quad \delta S_{2 \mu}=\Lambda_{1,1 \mu}+\partial_{\mu} \Lambda_{2} \quad, \quad \delta S_{2}=\Lambda_{2} k_{0}^{5} .
$$

where $<\lambda_{1} k_{1}^{\mu}>=\Lambda_{1,1}^{\mu}$ and $<\lambda_{2}>=\Lambda_{2}$.

In manifestly covariant notation the gauge transformation of $S_{1,1}^{\mu \nu}$ is modified to

$$
\delta S_{1,1 \mu \nu}=D_{(\mu} \Lambda_{1,1 \nu)} \quad, \quad \delta S_{2 \mu}=\Lambda_{1,1 \mu}+\partial_{\mu} \Lambda_{2} \quad, \quad \delta S_{2}=\Lambda_{2} k_{0}^{5} .
$$

while the others are unmodified.

It is easy to see that the equation (3.14) is not invariant under (3.16). The reason is not hard to find. The gauge transformation of the space time derivative of a field is usually taken to be the derivative of the gauge transformation of the field. But this is not what the loop variable prescription gives. Unfortunately, the prescription of the loop variable is the one that keeps the equation gauge invariant! As an example of this discrepancy consider the loop variable expression $L$

$$
L=k_{0 \rho} k_{1 \mu} k_{1 \nu}
$$


which is mapped to $S$, the covariant derivative of $S_{1,1 \mu \nu}$ :

$$
S=D_{\rho} S_{1,1 \mu \nu}
$$

Schematically we have the map

$$
\mathcal{M}: L \rightarrow S
$$

The gauge variation of $L$ is $L^{g}$

$$
\mathcal{G}: L \rightarrow L^{g}=L+\delta L=L+k_{0 \rho} \lambda_{1} k_{0(\mu} k_{1 \nu)}
$$

which leaves the spin $2 \mathrm{LV}$ equation invariant. However the change $\Delta S$ given by

$$
\begin{gathered}
\mathcal{M}: L^{g} \rightarrow S^{g}=S+\Delta S=S+<k_{0 \rho} \lambda_{1} k_{0(\mu} k_{1 \nu)}>= \\
S+D_{\rho} D_{(\mu} \Lambda_{1,1 \nu)}+\frac{1}{3}\left(R_{\nu \rho \mu}^{\alpha}+R_{\mu \rho \nu}^{\alpha}\right) \Lambda_{1,1 \alpha}
\end{gathered}
$$

is not the (covariant) derivative of the gauge variation of $S_{1,1 \mu \nu}$ : i.e. using (3.16) one expects that

$$
\mathcal{G}: S \rightarrow S+\delta S=S+D_{\rho} D_{(\mu} \Lambda_{1,1 \nu)}
$$

which is not equal to $S^{g}$ calculated above, because of the extra curvature coupling. In (3.14) there are two derivatives of the field $S^{\mu \nu}$ and this problem is more acute - one gets not only curvature terms but derivatives of the curvature.

\subsection{Imposing Gauge Invariance}

One can contemplate some ways out of this conflict. If we forcibly set $\delta S=\Delta S$ instead of using (3.19) we will have invariance of the equation. This would force us to vary the background gravitational field under a gauge transformation of the open string fields. This would mean that the covariant derivative does not commute with gauge transformations.

Another possibility is to change the action of $\mathcal{M}$ on $L$ (but not $\delta L$ ) so that $S$ is modified to $S^{\prime}=S+f$, but $\Delta S$ is the same. The we can set $\delta S^{\prime}=\delta S+\delta f=\Delta S$. If we can find such a tensor $f$ we are done. This means we do not modify the gauge transformation (3.16) but modify (3.17). This would mean modifying the space time equation of motion while keeping 
the loop equation unchanged. The gauge variation of the new space time equation under the conventional gauge variation will give all the additional curvature terms. Let us illustrate this in the example above by finding $f$.

Keeping in mind the fact that

$$
\delta\left(S_{2 \mu}-\frac{D_{\mu} S_{2}}{2 k_{0}^{5}}\right)=\Lambda_{1,1 \mu}
$$

we modify $S$ to

$$
S^{\prime}=S+f=D_{\rho} S_{\mu \nu}+\frac{1}{3}\left(R_{\nu \rho \mu}^{\alpha}+R_{\mu \rho \nu}^{\alpha}\right)\left(S_{2 \alpha}-\frac{D_{\alpha} S_{2}}{2 k_{0}^{5}}\right) \equiv F_{\rho \mu \nu}
$$

It is easy to see that $\delta S^{\prime}=\Delta S$. Thus we have found $f$. Thus if we say that $\mathcal{M}: L \rightarrow S^{\prime}$, we will find that we reproduce the variation (3.18) using the standard variations of the space time fields. In this way we have managed to reproduce the variation specified by the loop variable prescription and this guarantees the invariance of the equation.

This solution to the problem is easily generalized. In every case one modifies the map from loop variable expression to space time fields by adding some extra terms. These additional terms involve products of curvature tensor and auxiliary fields. These terms are chosen so that they have the required gauge variation. This is always possible because of relations of the form (3.20). Such relations can always be found for all the gauge parameters. This is because in a massive theory the gauge parameters are in one-to-one correspondence with auxiliary fields. The fact that there is an inverse power of $k_{0}^{5}$ in (3.20) (which gives the mass), tells you that it is crucial that we are dealing with a massive gauge field ${ }^{2}$. In string theory this is always the case for the higher spin fields, so this is not a problem. In the limit that the string tension goes to zero, the higher modes of the string become massless. In this case one should obtain a description of massless higher spin gauge fields in curved space.

We will now apply the above technique to the case at hand, viz. (3.8). Using the notation of the example above we let

$$
L=k_{0 \rho} k_{0 \sigma} k_{1 \mu} k_{1 \nu}
$$

We let

$$
\mathcal{M}: L\left(=k_{0 \rho} k_{0 \sigma} k_{1 \mu} k_{1 \nu}\right) \rightarrow S^{\prime}\left(=<k_{0 \rho} k_{0 \sigma} k_{1 \mu} k_{1 \nu}>\right)
$$

\footnotetext{
${ }^{2}$ This is reminiscent of the inverse power of the cosmological constant in higher spin massless gauge theories described in [15]
} 
It is a tensor $F$ given by

$$
S^{\prime}=F_{\rho \sigma \mu \nu}=D_{\rho} D_{\sigma} S_{\mu \nu}+\frac{1}{3}\left(R_{\mu \rho \sigma}^{\alpha}+R_{\sigma \rho \mu}^{\alpha}\right) S_{\alpha \nu}+\frac{1}{3}\left(R_{\nu \rho \sigma}^{\alpha}+R_{\sigma \rho \nu}^{\alpha}\right) S_{\mu \alpha}+f_{\rho \sigma \mu \nu}
$$

(We have supressed the subscripts " 1,1 " in both $S$ and $\Lambda$ below.) Then we evaluate

$$
\mathcal{M}: \delta L=<\lambda_{1} k_{0 \rho} k_{0 \sigma} k_{0(\mu} k_{1 \nu)}=\Delta S=G_{\rho \sigma \mu \nu}
$$

The exact expression for $G$ is given in the Appendix. The prescription is to choose $f$ such that

$$
\begin{aligned}
G_{\rho \sigma \mu \nu}= & D_{\rho} D_{\sigma} D_{(\mu} \Lambda_{\nu)}+\frac{1}{3}\left(R_{\mu \rho \sigma}^{\alpha}+R_{\sigma \rho \mu}^{\alpha}\right)\left(D_{\alpha} \Lambda_{\nu}+D_{\nu} \Lambda_{\alpha}\right)+ \\
& \frac{1}{3}\left(R_{\nu \rho \sigma}^{\alpha}+R_{\sigma \rho \nu}^{\alpha}\right)\left(D_{\alpha} \Lambda_{\mu}+D_{\mu} \Lambda_{\alpha}\right)+\delta f_{\rho \sigma \mu \nu}
\end{aligned}
$$

As an example, consider the term proportional to $D_{\nu} \Lambda_{\alpha}$. We find that

$$
\delta f_{\rho \sigma \mu \nu}=\left[-\frac{1}{6}\left(R_{\mu \rho \sigma}^{\alpha}+R_{\sigma \rho \mu}^{\alpha}\right)+\frac{1}{6} R_{\rho \sigma \mu}^{\alpha}\right] D_{\nu} \Lambda_{\alpha}
$$

This implies that we can take one of the terms in $f$ to be

$$
\left[-\frac{1}{6}\left(R_{\mu \rho \sigma}^{\alpha}+R_{\sigma \rho \mu}^{\alpha}\right)+\frac{1}{6} R_{\rho \sigma \mu}^{\alpha}\right] D_{\nu}\left(S_{2 \alpha}-D_{\alpha} \frac{S_{2}}{2 k_{0}^{5}}\right)
$$

This is $f_{\rho \sigma \mu \nu}^{1}$ of the Appendix.

Thus we calculate $F_{\rho \sigma \mu \nu}$ which is given in the Appendix. $F_{\rho \mu \nu}$ is given in (3.21). These in turn are determined by the two tensors $f_{\rho \mu \nu}$ and $f_{\rho \sigma \mu \nu}$. The corresponding tensor modification for terms involving $S_{2 \mu}$ and the scalar $S_{2}$ are zero.

In terms of these tensors the equations of motion are $\left(k_{0}^{5}=1\right)$ :

$$
\begin{gathered}
F_{\rho \mu \nu}^{\rho}+\left(k_{0}^{5}\right)^{2} S_{1,1}^{\mu \nu}-F_{\rho(\nu \mu)}{ }^{\rho}+F_{\mu \nu \rho}{ }^{\rho}+D_{(\mu} S_{2 \nu)}\left(k_{0}^{5}\right)^{2}-D_{(\mu} D_{\nu)} S_{2} k_{0}^{5}=0 \\
D^{\rho} D_{\rho} S_{2 \mu}-F_{\mu \rho}^{\rho}+F_{\mu \rho}{ }^{\rho}-D_{\mu} D^{\rho} S_{2 \rho}=0 \\
-F_{\rho \sigma}^{\rho \sigma}+F_{\rho \sigma}^{\rho \sigma}-\left(k_{0}^{5}\right)^{2} S_{1,1 \rho}^{\rho}-2 D_{\rho} S_{2}^{\rho}\left(k_{0}^{5}\right)^{2}-D^{\rho} D_{\rho} S_{2} k_{0}^{5}=0
\end{gathered}
$$

By construction they are gauge invariant under

$$
\delta S_{1,1 \mu \nu}=D_{(\mu} \Lambda_{1,1 \nu)} \quad, \quad \delta S_{2 \mu}=\Lambda_{1,1 \mu}+\partial_{\mu} \Lambda_{2} \quad, \quad \delta S_{2}=\Lambda_{2} k_{0}^{5} .
$$

The same procedure can clearly be applied to spin $3^{3}$ and the higher modes also.

\footnotetext{
${ }^{3}$ The LV equations for spin 3 are given in [5]
} 


\section{Conclusions}

We have given a method of writing down gauge and generally covariant equations of motion for the higher spin modes of the open bosonic string. Explicit equations for spin 2 are given (3.28) as an illustration of the method . The method is a generalization of the flat space method of [5]. The main point of departure, apart form the obvious one of covariantizing, is that the map from loop varables to space time fields is modified. The modification is designed to reproduce the gauge transformation of the loop variable. This ensures that the equations are gauge invariant. The modification involves adding terms that involve inverse power of the mass of the higher string modes. Since the mass is proportional to the string tension, it would be interesting to try and obtain the massless case as a limit of vanishing string tension.

The loop variable method in the flat space case was easily generalized to the interacting case by thickening the loop to a band [7]. If this holds in curved space also then we have a set of interacting higher spin massive fields in curved space. This can then be treated as a step towards a proposal for a manifestly background independent formulation of string theory[22].

Acknowledgements: I would like to thank G. Date and S. Kalyana Rama for reading the manuscript and giving some useful comments.

\section{A Appendix: Calculation of $F$.}

In this Appendix we calculate the tensor $F_{\rho \sigma \mu \nu}$. The first step is to calculate $G_{\rho \sigma \mu \nu}$ defined by

$$
G_{\rho \sigma \mu \nu}=<\lambda_{1} k_{0 \rho} k_{0 \sigma} k_{0(\mu} k_{0 \nu)}>
$$

We have defined this as $\left.\partial_{\rho} \partial_{\sigma} \partial_{\mu} \Lambda_{\nu}(0)\right)\left(\partial_{\mu}=\frac{\partial}{\partial y^{\mu}}\right)$ written in covariantised form. We use the Taylor expansion (2.7) to write

$$
\begin{gathered}
\Lambda_{\nu}(y)=\Lambda_{\nu}(0)+y^{\alpha} D_{\alpha} \Lambda_{\nu}+\frac{y^{\alpha} y^{\beta}}{2 !}\left(D_{\alpha} D_{\beta} \Lambda_{\nu}+\frac{1}{3} R_{\beta \alpha \nu}^{\sigma} \Lambda_{\sigma}\right)+ \\
\frac{y^{\alpha} y^{\beta} y^{\gamma}}{3 !}\left(D_{\alpha} D_{\beta} D_{\gamma} \Lambda_{\nu}-R_{\alpha \nu \beta}^{\sigma} D_{\gamma} \Lambda_{\sigma}-\frac{1}{2} D_{\alpha} R_{\beta \nu \gamma}^{\sigma} \Lambda_{\sigma}\right)+\ldots
\end{gathered}
$$

Acting on this with $\partial_{\rho} \partial_{\sigma} \partial_{\mu}$ gives

$$
\frac{1}{3 !}\left(D_{(\rho} D_{\sigma} D_{\mu)} \Lambda_{\nu}-R_{(\rho \nu \sigma}^{\alpha} D_{\mu)} \Lambda_{\alpha}-\frac{1}{2} D_{(\rho} R_{\sigma \nu \mu)}^{\alpha} \Lambda_{\alpha}\right)
$$


where the symmetrization is over the three indices $\rho, \sigma, \mu$.

We denote the three terms as I,II and III.

I:

The first term has to brought into the form $D_{\rho} D_{\sigma} D_{\mu}$. This can be achieved by using the commutation rules:

$$
\begin{gathered}
{\left[D_{\rho}, D_{\sigma}\right] W_{\mu}=R_{\mu \sigma \rho}^{\alpha} W_{\alpha}} \\
{\left[D_{\rho}, D_{\sigma}\right] W_{\mu \nu}=R_{\mu \sigma \rho}^{\alpha} W_{\alpha \nu}+R_{\nu \sigma \rho}^{\alpha} W_{\mu \alpha}}
\end{gathered}
$$

If we do this and symmetrize on $\mu, \nu$ we get

$$
\begin{gathered}
G_{\rho \sigma \mu \nu}^{I}=D_{\rho} D_{\sigma} D_{(\mu} \Lambda_{\nu)}+\frac{1}{2}\left(D_{\rho}\left(R_{\nu \sigma \mu}^{\alpha}+R_{\mu \sigma \nu}^{\alpha}\right)\right) \Lambda_{\alpha} \\
+\frac{1}{6}\left(D_{\mu} R_{\nu \sigma \rho}^{\alpha}+D_{\nu} R_{\mu \sigma \rho}^{\alpha}\right) \Lambda_{\alpha}+\frac{1}{6}\left(D_{\sigma}\left(R_{\nu \rho \mu}^{\alpha}+R_{\mu \rho \nu}^{\alpha}\right)\right) \Lambda_{\alpha} \\
+\frac{1}{2}\left(R_{\nu \sigma \mu}^{\alpha}+R_{\mu \sigma \nu}^{\alpha}\right) D_{\rho} \Lambda_{\alpha}+\frac{1}{6}\left(R_{\nu \rho \sigma}^{\alpha} D_{\mu} \Lambda_{\alpha}+R_{\mu \rho \sigma}^{\alpha} D_{\nu} \Lambda_{\alpha}\right) \\
+\frac{1}{2}\left(R_{\nu \rho \mu}^{\alpha}+R_{\mu \rho \nu}^{\alpha}\right) D_{\sigma} \Lambda_{\alpha}+\frac{1}{3}\left(R_{\sigma \rho \mu}^{\alpha}+R_{\mu \rho \sigma}^{\alpha}\right) D_{\alpha} \Lambda_{\nu} \\
+\frac{1}{3}\left(R_{\sigma \rho \nu}^{\alpha}+R_{\nu \rho \sigma}^{\alpha}\right) D_{\alpha} \Lambda_{\mu}
\end{gathered}
$$

II:

The second term on symmetrization gives:

$$
\begin{gathered}
G_{\rho \sigma \mu \nu}^{I I}=-\frac{1}{3 !}\left[\left(R_{\rho \nu \sigma}^{\alpha}+R_{\sigma \nu \rho}^{\alpha}\right) D_{\mu} \Lambda_{\alpha}+\left(R_{\rho \mu \sigma}^{\alpha}+R_{\sigma \mu \rho}^{\alpha}\right) D_{\nu} \Lambda_{\alpha}\right. \\
\left.\quad+\left(R_{\mu \nu \rho}^{\alpha}+R_{\nu \mu \rho}^{\alpha}\right) D_{\sigma} \Lambda_{\alpha}+\left(R_{\mu \nu \sigma}^{\alpha}+R_{\nu \mu \sigma}^{\alpha}\right) D_{\rho} \Lambda_{\alpha}\right]
\end{gathered}
$$

III:

The third term gives

$$
\begin{aligned}
G_{\rho \sigma \mu \nu}^{I I I} & =-\frac{1}{12}\left[D_{\rho} R_{\mu \nu \sigma}^{\alpha}+D_{\rho} R_{\nu \mu \sigma}^{\alpha}+D_{\mu} R_{\rho \nu \sigma}^{\alpha}+D_{\nu} R_{\rho \mu \sigma}^{\alpha}\right. \\
& \left.+D_{\mu} R_{\sigma \nu \rho}^{\alpha}+D_{\nu} R_{\sigma \mu \rho}^{\alpha}+D_{\sigma} R_{\mu \nu \rho}^{\alpha}+D_{\sigma} R_{\nu \mu \rho}^{\alpha}\right]
\end{aligned}
$$

Adding everything gives

$$
G_{\rho \sigma \mu \nu}=D_{\rho} D_{\sigma} D_{(\mu} \Lambda_{\nu)}+\frac{2}{3}\left(R_{\nu \sigma \mu}^{\alpha}+R_{\mu \sigma \nu}^{\alpha}\right) D_{\rho} \Lambda_{\alpha}+\frac{2}{3}\left(R_{\nu \rho \mu}^{\alpha}+R_{\mu \rho \nu}^{\alpha}\right) D_{\sigma} \Lambda_{\alpha}+
$$




$$
\begin{gathered}
{\left[\frac{1}{6}\left(R_{\nu \rho \sigma}^{\alpha}+R_{\rho \sigma \nu}^{\alpha}+R_{\sigma \rho \nu}^{\alpha}\right)\right] D_{\mu} \Lambda_{\alpha}+\left[\frac{1}{6}\left(R_{\mu \rho \sigma}^{\alpha}+\left(R_{\rho \sigma \mu}^{\alpha}+R_{\sigma \rho \mu}^{\alpha}\right)\right] D_{\nu} \Lambda_{\alpha}+\right.} \\
\frac{1}{3}\left(R_{\sigma \rho \mu}^{\alpha}+R_{\mu \rho \sigma}^{\alpha}\right) D_{\alpha} \Lambda_{\nu}+\frac{1}{3}\left(R_{\sigma \rho \nu}^{\alpha}+R_{\nu \rho \sigma}^{\alpha}\right) D_{\alpha} \Lambda_{\mu}+ \\
\frac{7}{12}\left(D_{\rho} R_{\mu \sigma \nu}^{\alpha}+D_{\rho} R_{\nu \sigma \mu}^{\alpha}\right) \Lambda_{\alpha}+\frac{1}{4}\left(D_{\sigma} R_{\mu \rho \nu}^{\alpha}+D_{\sigma} R_{\nu \rho \mu}^{\alpha}\right) \Lambda_{\alpha}+ \\
\left(-\frac{1}{4} D_{\mu} R_{\rho \nu \sigma}^{\alpha}+\frac{1}{12} D_{\mu} R_{\sigma \nu \rho}^{\alpha}\right) \Lambda_{\alpha}+ \\
\left(-\frac{1}{4} D_{\nu} R_{\rho \mu \sigma}^{\alpha}+\frac{1}{12} D_{\nu} R_{\sigma \mu \rho}^{\alpha}\right) \Lambda_{\alpha}
\end{gathered}
$$

We have to set this equal to

$$
D_{\rho} D_{\sigma} \delta S_{\mu \nu}+\frac{1}{3}\left(R_{\mu \rho \sigma}^{\alpha}+R_{\sigma \rho \mu}^{\alpha}\right) \delta S_{\alpha \nu}+\frac{1}{3}\left(R_{\nu \rho \sigma}^{\alpha}+R_{\sigma \rho \nu}^{\alpha}\right) \delta S_{\mu \alpha}+\delta f_{\rho \sigma \mu \nu}
$$

Comparing both sides we can write $f$ as the sum of five terms:

$$
f_{\rho \sigma \mu \nu}=\sum_{i=1}^{5} f_{\rho \sigma \mu \nu}^{i}
$$

with:

$$
\begin{gathered}
f_{\rho \sigma \mu \nu}^{1}=-\frac{1}{6}\left(R_{\mu \rho \sigma}^{\alpha}+R_{\sigma \rho \mu}^{\alpha}+R_{\rho \sigma \mu}^{\alpha}\right) D_{\nu}\left(S_{2 \alpha}-\frac{D_{\alpha} S_{2}^{5}}{2 k_{0}^{5}}\right) \\
f_{\rho \sigma \mu \nu}^{2}=-\frac{1}{6}\left(R_{\nu \rho \sigma}^{\alpha}+R_{\sigma \rho \nu}^{\alpha}+R_{\rho \sigma \nu}^{\alpha}\right) D_{\mu}\left(S_{2 \alpha}-\frac{D_{\alpha} S_{2}^{5}}{2 k_{0}^{5}}\right) \\
f_{\rho \sigma \mu \nu}^{3}=\frac{2}{3}\left(R_{\nu \rho \mu}^{\alpha}+R_{\mu \rho \nu}^{\alpha}\right) D_{\sigma}\left(S_{2 \alpha}-\frac{D_{\alpha} S_{2}^{5}}{2 k_{0}^{5}}\right) \\
f_{\rho \sigma \mu \nu}^{4}=\frac{2}{3}\left(R_{\nu \sigma \mu}^{\alpha}+R_{\mu \sigma \nu}^{\alpha}\right) D_{\rho}\left(S_{2 \alpha}-\frac{D_{\alpha} S_{2}^{5}}{2 k_{0}^{5}}\right) \\
f_{\rho \sigma \mu \nu}^{5}=\left[\frac{7}{12}\left(D_{\rho} R_{\mu \sigma \nu}^{\alpha}+D_{\rho} R_{\nu \sigma \mu}^{\alpha}\right)+\frac{1}{4}\left(D_{\sigma} R_{\mu \rho \nu}^{\alpha}+D_{\sigma} R_{\nu \rho \mu}^{\alpha}\right)+\right. \\
\left(-\frac{1}{4} D_{\mu} R_{\rho \nu \sigma}^{\alpha}+\frac{1}{12} D_{\mu} R_{\sigma \nu \rho}^{\alpha}\right)+ \\
\left.\left(-\frac{1}{4} D_{\nu} R_{\rho \mu \sigma}^{\alpha}+\frac{1}{12} D_{\nu} R_{\sigma \mu \rho}^{\alpha}\right)\right]\left(S_{2 \alpha}-\frac{D_{\alpha} S_{2}^{5}}{2 k_{0}^{5}}\right)
\end{gathered}
$$

Thus we finally get

$$
F_{\rho \sigma \mu \nu}=D_{\rho} D_{\sigma} S_{\mu \nu}+\frac{1}{3}\left(R_{\mu \rho \sigma}^{\alpha}+R_{\sigma \rho \mu}^{\alpha}\right) S_{\alpha \nu}+\frac{1}{3}\left(R_{\nu \rho \sigma}^{\alpha}+R_{\sigma \rho \nu}^{\alpha}\right) S_{\mu \alpha}+f_{\rho \sigma \mu \nu}
$$




\section{B Appendix: Loop Variables}

We give a short summary of the loop variable approach here. The basic idea introduced in [5] is to generalize the vertex operators of the sigma model by introducing additional variables $x_{n}, n>0$ that, very roughly, have the

property that $\frac{\partial}{\partial x_{n}} \approx \partial_{z}^{n}$. They can be thought of as the modes of an einbein that make the loop variable diffeomorphism invariant.

We thus consider the following loop variable:

$$
e^{i \int_{c} \alpha(s) k(s) \partial_{z} X(z+s) d s+i k_{0} X(z)}
$$

It is understood that $Y_{0}=Y . \alpha(s)$ is an einbein. Let us assume the following Laurent expansion:

$$
\alpha(s)=1+\frac{\alpha_{1}}{s}+\frac{\alpha_{2}}{s^{2}}+\frac{\alpha_{3}}{s^{3}}+\ldots
$$

Let us define

$$
\begin{aligned}
Y & =X+\alpha_{1} \partial_{z} X+\alpha_{2} \partial_{z}^{2} X+\alpha_{3} \frac{\partial_{z}^{3} X}{2}+\ldots+\frac{\alpha_{n} \partial_{z}^{n} X}{(n-1) !}+\ldots \\
& =X+\sum_{n>0} \alpha_{n} \tilde{Y}_{n} \\
Y_{1} & =\partial_{z} X+\alpha_{1} \partial_{z}^{2} X+\alpha_{2} \frac{\partial_{z}^{3} X}{2}+\ldots+\frac{\alpha_{n-1} \partial_{z}^{n} X}{(n-1) !}+\ldots \\
\ldots & \cdots \\
Y_{m} & =\frac{\partial_{z}^{m} X}{(m-1) !}+\sum_{n>m} \frac{\alpha_{n-m} \partial_{z}^{n} X}{(n-1) !}
\end{aligned}
$$

If we define $\alpha_{0}=1$ then the $>$ signs in the summations above can be replaced by $\geq$.

Using these equations one can write

$$
e^{i \int_{c} \alpha(s) k(s) \partial_{z} X(z+s) d s+i k_{0} X(z)}=e^{i \sum_{n \geq 0} k_{n} Y_{n}(z)}
$$

It is understood that $Y_{0}=Y$.

Let us now introduce $x_{n}$ by the following:

$$
\alpha(s)=\sum_{n \geq 0} \alpha_{n} s^{-n}=e^{\sum_{m \geq 0} s^{-m} x_{m}}
$$


Thus

$$
\begin{aligned}
\alpha_{1} & =x_{1} \\
\alpha_{2} & =\frac{x_{1}^{2}}{2}+x_{2} \\
\alpha_{3} & =\frac{x_{1}^{3}}{3 !}+x_{1} x_{2}+x_{3}
\end{aligned}
$$

They satisfy the property,

$$
\frac{\partial \alpha_{n}}{\partial x_{m}}=\alpha_{n-m}, \quad n \geq m
$$

Using this we see that

$$
Y_{n}=\frac{\partial Y}{\partial x_{n}}
$$

Now we can impose conformal invariance by demanding $\frac{\delta}{\delta \sigma}=0$ where $\sigma$ is the Liouville mode : $\langle X(z) X(z)\rangle=\ln a+\sigma$ ( $a$ is the world sheet cutoff). Except we will define $\Sigma=\langle Y(z) Y(z)\rangle$. This is equal to the previous $\sigma$ in coordinates where $\alpha(s)=1$. Thus we have for the coincident two point functions:

$$
\begin{aligned}
\langle Y Y\rangle & =\Sigma \\
\left\langle Y_{n} Y\right\rangle & =\frac{1}{2} \frac{\partial \Sigma}{\partial x_{n}} \\
\left\langle Y_{n} Y_{m}\right\rangle & =\frac{1}{2}\left(\frac{\partial^{2} \Sigma}{\partial x_{n} \partial x_{m}}-\frac{\partial \Sigma}{\partial x_{n+m}}\right)
\end{aligned}
$$

Using this the normal ordering gives:

$$
\begin{aligned}
e^{i \int_{c} \alpha(s) k(s) \partial_{z} X(z+s) d s+i k_{0} X(z)=} & e^{i \sum_{n \geq 0} k_{n} Y_{n}(z)} \\
= & \exp \left\{k_{0}^{2} \Sigma+\sum_{n>0} k_{n} \cdot k_{0} \frac{\partial \Sigma}{\partial x_{n}}+\right. \\
& \left.\sum_{n, m>0} k_{n} \cdot k_{m} \frac{1}{2}\left(\frac{\partial^{2} \Sigma}{\partial x_{n} \partial x_{m}}-\frac{\partial \Sigma}{\partial x_{n+m}}\right)\right\} \\
& : e^{i \sum_{n \geq 0} k_{n} Y_{n}(z)}:
\end{aligned}
$$

We can now operate with $\frac{\delta}{\delta \Sigma}$ and set $\Sigma=0$. We will only give one sample variation here: 
$\frac{\delta}{\delta \Sigma}\left[k_{n} \cdot k_{m} \frac{1}{2}\left(\frac{\partial^{2} \Sigma}{\partial x_{n} \partial x_{m}}-\frac{\partial \Sigma}{\partial x_{n+m}}\right)\right]: e^{i k_{0} . Y}:=:\left(\frac{1}{2} i k_{0}^{\mu} i k_{0}^{\nu} Y_{n}^{\mu} Y_{m}^{\nu}+i k_{0}^{\mu} Y_{n+m}^{\mu}\right) e^{i k_{0} \cdot Y}:$

One can thus collect all the coefficients of a particular vertex operator, say : $Y_{n}^{\mu} e^{i k_{0} . Y}$ :, and this gives the free equation of motion. Note that they never contain more than two space-time derivatives.

We turn to the issue of gauge invariance. We have assumed that $\alpha(s)$ is being integrated over, which is why we are allowed to integrate by parts. This means that

$$
k(s) \rightarrow \lambda(s) k(s)
$$

is equivalent to $\alpha(s) \rightarrow \lambda(s) \alpha(s)$, which is clearly just a change of an integration variable. Assuming the measure is invariant this does nothing to the integral. The measure $\mathcal{D} \alpha(s)$ has been chosen to be $\prod_{n} d x_{n}$. If we set $\lambda(s)=e^{\sum_{m} y_{m} s^{-m}}$, then the gauge transformation (B.13) is just a translation, $x_{n} \rightarrow x_{n}+y_{n}$ which leaves the measure invariant. Thus we conclude that (B.13) gives the gauge transformation.

If we expand $\lambda(s)$ in inverse powers of $s$

$$
\lambda(s)=\sum_{n} \lambda_{n} s^{-n}
$$

Then we can write (B.13) as

$$
k_{n} \rightarrow \sum_{m=0}^{n} \lambda_{m} k_{n-m}
$$

We set $\lambda_{0}=1$.

In order to interpret these equations in terms of space-time fields we use (??). They have to be extended to include $\lambda$. Thus we assume that the string wave-functional is also a functional of $\lambda(s)$. Thus we set

$$
\begin{aligned}
\left\langle\lambda_{1}\right\rangle & =\Lambda_{1}\left(k_{0}\right) \\
\left\langle\lambda_{1} k_{1}^{\mu}\right\rangle & =\Lambda_{11}^{\mu}\left(k_{0}\right) \\
\left\langle\lambda_{2}\right\rangle & =\Lambda_{2}\left(k_{0}\right)
\end{aligned}
$$

The gauge transformations (B.14) thus become on mapping to space time fields by evaluating $\langle.$.$\rangle :$ 


$$
\begin{aligned}
A^{\mu}\left(k_{0}\right) & \rightarrow A^{\mu}(k o)+k_{0}^{\mu} \Lambda_{1}\left(k_{0}\right) \\
S_{2}^{\mu}\left(k_{0}\right) & \rightarrow S_{2}^{\mu}(k o)+k_{0}^{\mu} \Lambda_{2}\left(k_{0}\right)+\Lambda_{11}^{\mu} \\
S_{11}^{\mu \nu} & \rightarrow S_{11}^{\mu \nu}+k_{0}^{(\mu} \Lambda_{11}^{\nu)}
\end{aligned}
$$

These are more or less the canonical gauge transfomations for a massive spin two field. ${ }^{4}$ Now it is known that the gauge transformation parameters of higher spin fields obey a certain tracelessness condition [16, 17]. We will see this below also.

When one actually performs the gauge transformations we find the following mechanism for gauge invariance. It changes the normal ordered loop variable by a total derivative in $x_{n}$ which doesn't affect the equation of motion. More precisely the gauge variation of the loop variable is a term of the form $\frac{d}{d x_{n}}[A(\Sigma) B]$, where $B$ doesn't depend on $\Sigma$. The coefficient of $\delta \Sigma$ is obtained as

$$
\begin{gathered}
\int \delta\left(\frac{d}{d x_{n}}[A(\Sigma) B]\right)=\int\left(\frac{d}{d x_{n}}\left(\frac{\delta A}{\delta \Sigma} \delta \Sigma\right) B+\frac{\delta A}{\delta \Sigma} \delta \Sigma \frac{d B}{d x_{n}}\right) \\
=\int\left[-\frac{\delta A}{\delta \Sigma} \frac{d B}{d x_{n}}+\frac{\delta A}{\delta \Sigma} \frac{d B}{d x_{n}}\right] \delta \Sigma=0
\end{gathered}
$$

Here we have used an integration by parts.

Actually one finds on explicit calculation that the variation is not a total derivative. This is because in deriving (B.11) some identities have been used. Thus only if we use those identities in the variation will we be able to write the variation as a total derivative. However we do not want to use them because we would like to leave $\Sigma$ unconstrained when we vary. Thus constraints have to be imposed elsewhere. It can easily be checked that the terms that have to be put to zero are all of the form

$$
\lambda_{n} k_{m} \cdot k_{p} \cdots
$$

where $\ldots$ refers to any other factors of $k_{m}[5,18]$. Thus all traces of gauge parameters have to be set to zero. This thus explains the tracelessness mentioned earlier.

In $[5,18]$ some examples, namely spin-2 and spin-3 are explicitly worked out.

\footnotetext{
${ }^{4}$ They become identical after we perform a dimensional reduction. This will be described later.
} 


\section{References}

[1] C. Lovelace, Phys. Lett. B135,75 (1984).

[2] C. Callan, D. Friedan, E. Martinec and M. Perry, Nucl. Phys. B262,593 (1985).

[3] A. Sen, Phys. Rev. D32,2102 (1985).

[4] E. Fradkin and A.A. Tseytlin, Phys. Lett. B151,316 (1985).

[5] B. Sathiapalan, Nucl. Phys. B326, (1989) 376.

[6] C. S. Aulakh and D. Sahdev, Phys. Lett. B164, (1985) 293; Phys. Lett. B173, (1986) 241.

[7] B. Sathiapalan, Int.J.Mod.Phys. A18 (2003) 767, hep-th/0207098.

[8] B. Sathiapalan, Mod. Phys. Lett. A19 (2004) 59, hep-th/0310128.

[9] B. Sathiapalan, hep-th/0405119.

[10] C. Aragone and S. Deser, Phys. Lett. B86 (1979) 161.

[11] A. Bengtsson, I. Bengtsson and L. Brink, Nucl. Phys. B227 (1983) 31,41 .

[12] F. A. Berends, G. J. Burgers and H. van Dam, Z. Phys. C24 (1984) 247

[13] I. L. Buchbinder, D. M. Gitman, V. A. Krykhtin, V. D. Pershin, Nucl. Phys. B584 (2000) 615, hep-th/9910188.

[14] E. S. Fradkin and M. A. Vasiliev, Phys. Lett. B189 (1987) 89; Nucl. Phys. B291 (1987) 141.

[15] M. A. Vasiliev, hep-th/0409260 and references therein.

[16] C. Fronsdal, Phys. Rev. D18 (1978) 3624.

[17] L. P. S. Singh and C. R. Hagen, Phys. Rev. D9 (1974) 898.

[18] B. Sathiapalan, hepth/9207051; Nucl. Phys. B405 (1993) 367.

[19] A. Z. Petrov, "Einstein Spaces", Pergamon Press, Oxford, 1969. 
[20] L. Alvarez-Gaume, D. Z. Freedman and S. Mukhi, Ann. Phys. 134, (1981) 85.

[21] D. Friedan, Ann. Phys. 163, (1985) 318.

[22] E. Witten, Phys. Rev. D46 (1992)5467. 